sections on anatomy and physiology; the evaluation of the dizzy patient - an analysis of history, examination and laboratory tests and the diagnosis and treatment of common neuro-otologic disorders. The last is disappointingly brief and in many ways uncritical, and old fashioned. By contrast, the first two sections are detailed, precise and provide a most helpful current account of clinically relevant anatomy and investigation. MRI, evoked potential techniques, electronystagmography and the newer rotational investigations of semicircular canal function are considered and well illustrated.

I think this a useful book, which attempts and to a large measure succeeds in illuminating a difficult, tenebrous area of neurology. It is not cumbersome in size or content and will certainly commend it to colleagues and junior staff in training.

JMS PEARCE

Neuroendocrinological Aspects of Neurosurgery. Proceedings of the Third Advanced Seminar in Neurosurgical Research, Venice April 30-May 11987. Edited by J D PICKARD, F COHADON, J LOBO Antunes. (Pp 128; Price: DM158.) Wien, Springer-Verlag, 1990. ISBN 3-211-82160-

Under the guidance of its recent and current chairmen, the Research committee of the European Association of Neurological Surgeons, with the support of Fidia, has held a series of very successful meetings, directed at younger staff neurosurgeons and senior trainees with an active involvement in research, designed to link selected areas of clinical neurosurgery with basic neuroscience. This book is the proceedings of the third (1987) meeting.

The first half of the book covers anatomy, physiology, neurohumoral and clinical aspects of the hypothalamo-pituitary axis. Everitt and Hökfelt provide an authoritative description of the anatomy of the hypothalamus and its connections. They illustrate the wealth of information now available on projections, receptors, and neurotransmitter co-existence. An attempt is made to integrate some of this material in the chapter by Vincent and Simonnet which follows. They emphasise the analogies between classical neurotransmission and secretion, and the coupling of behavioural and neuroendocrine homeostatic mechanisms, for example drinking and vasopressin secretion in response to osmotic stimulation. Some newer concepts are also developed, such as dendritic liberation of neurotransmitters, and autoreceptors. The chapters by Page et al and Teasdale $e t$ al are excellent reviews of the medical and surgical aspects of hypothalamic and pituitary diseases and their treatment.

However, it is the group of chapters on the relationships of the CNS with systemic metabolism and fluid balance in subarachnoid haemorrhage and head injury which give this book much broader relevance, for physicians as well as for surgeons. There are excellent and very lucid reviews of CNS control of fluid balance, and its disturbances in neurosurgical patients, by Lightman and Walker respectively. The chapters by NeilDwyer, Wijdicks, Nelson and Doczi and their respective collaborators are essential reading for vascular neurosurgeons and neurologists.
The use of double column A4 format provides for a large volume of information within 128 pages, and results in a conveniently thin book which will slip easily into a briefcase. It is an excellent expression of the collective strengths of European clinica neuroscience, and the price makes it a good buy for a departmental library. Potentia specialty fellowship candidates-and examiners - may well find it useful. ANTHONY STRONG

Conceptual Issues in Psychological Medicine. By MICHAEL SHEPHERD. (Pp 335 Price £40.00) London, Routledge, 1990. ISBN 0-415-03727-1.

The title of this book is misleading. Professor Shepherd has already published two volumes of his collected papers and this third volume contains a motley collection of papers, essays and commentaries that for one reason, or another were unsuitable for inclusion in either of the earlier collections. A hundred pages, no less, are occupied by case histories of morbid jealousy and half the other eighteen articles are brief essays of less than five thousand words. All the same they make compelling bedside reading, for Michael Shepherd is always thoughtful and perceptive with a fluent pen, a well developed sense of history and a keen eye for the shortcomings of contemporary Anglo American orthodoxies. $\mathrm{He}$ is also impressively erudite and, like Macaulay, Aldous Huxley and his mentor Aubrey Lewis, delights in trailing his learning before his readers. The paper on morbid jealousy is, despite its impedimenta, a classical clinical description and there are important messages for contemporary psychiatry in several of the historical and literary pieces.

R E KENDELL

\section{Immunologic Mechanisms in} Neurologic and Psychiatric Disease. (Research Publications: Association for Research in Nervous and Mental Disease, Vol. 68) Edited by BYRON H. WAKSMAN. (Pp 336; Price \$144.00) New York, Raven Press, 1990. ISBN 0-88167-593-8.

This volume admirably maintains the high standard of its numerous predecessors dating from 1920. In view of the large number of books on neuroimmunology which have appeared in recent years, it is always somewhat daunting to have to confront yet another volume on this subject. However, in this case the effort is certainly worthwhile since this book provides a large number of high quality reviews all of which display a uniformly consistent standard of discussion and presentation. The two broad messages which came over to me after reading this were firstly, that basic mechanisms in immunology are being recognised as being increasingly complex in nature which in turn is reflected by the increasing sophistication of the concepts and techniques applied to neuroimmunological problems; and secondly, that the evidence for a close association between the immune systems and the CNS is increasing at a rapid rate.

The book is divided into several sections which follow each other in a logical and coherent sequence. The opening chapters cover basic neuroimmunological mechanisms with excellent and thoughtful overviews on the genetic, molecular and general aspects of neuroimmunology. In the second section there are concise reviews on the immunopathological mechanisms of Multiple Sclerosis, noninflammatory immune mechanisms of CNS diseases and nervous system-immune system interactions. The third section covers monophasic autoimmune inflammatory diseases of the nervous system, chronic demyelinating diseases, tropical spastic paraparesis and the topical subject of HTLV-1 myelopathy, intraocular inflammatory disease and immune aspects of inflammatory myopathies.

I found the subsequent section on immunity and psychiatric disorders particularly interesting, covering the relationship between autoimmunity and depression, brain and behaviour and the immune system in general as well as neurological aspects of SLE. Non-inflammatory autoimmune diseases are then considered including very useful reviews of paraneoplastic syndromes, monoclonal gamopathies and neurological syndromes, disorders of the myoneural junction and motor neurone disease. The final chapters cover the subject of virus infection in $T$-cell deficient patients including SSPE, PML, a particularly thoughtful discussion of the pathogenesis of the AIDS dementia complex, behavioural correlates of HIV infection and finally the immunology of transmissible spongiform encephalopathy.

I found this book informative, stimulating, concise and full of original ideas. The production is excellent, the illustrations good and the referencing comprehensive. It is certainly not easy reading for the clinician without a working knowledge of neuroimmunology and it is also very expensive. Nevertheless, I shall find this volume very useful indeed and would certainly recommend that all good neurological and general medical libraries acquire it. Researchers in the field of neuroimmunology as well as many other clinicians and scientists would also find it to be a valuable aid to their work.

P G E KENNEDY

Cerebral Blood Flow and Metabolism Physiological Society Study Guides. BY A MURRAY HARPER AND SHEILA JENNETT. (Pp 110; Price: $£ 22.50$ hb; $£ 7.95$ pb). 1990, Manchester University Press. ISBN 0-71902892-2 hb, 2893-0 pb.

This concise, multi-author text is the latest in a series of Physiological Society Study Guides and is based on a teaching symposium on cerebral blood flow and metabolism, held in Glasgow in 1987, and updated to take into account recent developments. It is primarily aimed as an introductory text for those starting out in this increasingly complex field and is not intended as a research review for the seasoned investigator.

The five chapters: physiological control, innervation of the cerebral vasculature, cerebrovascular reactivity, measurement and anatomy of cerebral blood flow and the tomographic methods are clearly presented, with a useful review of important literature and supplemented by the frequent use of complementary figures. In this respect, the aims of the book have been achieved and I was 
particularly pleased to see that the mathematical derivations were simple to follow and did not instill the usual feeling of ignorance and despair!

This book is to be recommended to those starting out in this field and should provide enough information and important references to guide the reader to the more complex reference text-books. The hardback price $(£ 22.50)$ may be slightly off-putting for such a short text, but the provision of a paperback edition $(£ 7.95)$ should make it very much more attractive.

Two or three relatively minor points became apparent on reviewing the book. I think it would have been more appropriate to have put the chapter on anatomy and methods for measuring CBF after the chapter on innervation and follow this with an explanation of the tomographic methods. The chapter on the merits of evaluating cerebrovascular reactivity is well presented, but should have been at the end. Some chapters included references to various papers, but it was not always clear as to which author was being cited. The section on tomographic methods did not include any reference to Xenon-CT and included less than half a page on Single Photon Emission Computed Tomography and as a consequence was too brief and did not really explain the principles and methodology of this now well used technique. Finally, a simple table comparing the relative costs of each of the methods of measuring $\mathrm{CBF}$, together with examination times, radiation risk and their principal advantages and limitations would have been very useful.

A ROSS NAYLOR

A History of Stroke: Its Recognition and Treatment. By WM.S. FIELDS AND NA LEMAK. (Pp 211; Price $£ 30.00$ ). 1989, Oxford University Press. ISBN 0-19-505755-4.

This history of stroke opens with the Greeks and travels from them to 1915 in 28 pages a large part of which is taken up with illustrations and references. There follow a series of chapters on collateral circulation, arteriography, surgery, medical management, hypertension and epidemiology. Each of these consists largely of a list of the important papers with a brief account of what they contained. There is no attempt to explain how these various developments interacted, what triggered advances, why important discoveries were sometimes ignored for decades. Still less is there any account of the great controversies which marked progress in the management of cerebrovascular disease. As a bibliography this book is useful; as a history it fails.

JOHN MARSHALI

Systemic Pathology, Vol. 4 3rd Edition, Nervous System, Muscle and Eyes. Edited by wSTC SYMMERS AND RO WELLER. (Pp 776; Illustrated; Price: $£ 97 \cdot 50$ ). Edinburgh, Churchill Livingstone, 1990. ISBN 0-44303312-9.

This large textbook is part of the well-respected series "Systemic Pathology", a collection of monographs. In Volume 4 Professor Weller brings together contributions from some of the most senior neuropathologists in Britain to produce a textbook for histopathologists and an up-to-date source of information on all aspects of neuropathology for clinicians and basic scientists.

The book is divided into four parts dealing with central nervous system, peripheral nervous system, muscle and eyes and each begins with an account of normal anatomy and general reactions of the tissue to disease. This is particularly helpful to set the scene for readers with a background in general pathology. These readers will also benefit from chapter three, Pathophysiology of Raised Intracranial Pressure, which highlights some of the specific reactions of the central nervous system which result from its uniquely protected environment.

There is good practical advice on handling tissues of the nervous system in the first section, but neither here, nor in the section on muscle disease is the reader given specific guidance on handling a fresh muscle biopsy, where inappropriate fixation can make the sample useless for diagnosis. Use of histological stains and immunocytochemical markers are discussed in the first section with further reference to these methods throughout subsequent chapters dealing with specific diseases.

In the section on the central nervous system two chapters would seem to appeal to clinicians in particular. The chapter on Epilepsy deals in depth with the pathology associated with epilepsy and pathogenesis of epileptic brain damage although avoiding more than a superficial confrontation with the pathology of symptomatic epilepsy. Also of particular relevance to clinicians is "Ageing and the Dementias". This subject currently commands much attention. However, here the changes of normal ageing are compared with those seen in the dementias in an interesting and tightly written chapter.

It has always surprised me that brain tumours are not discussed in the standard neuropathology texts. In line with other volumes in the series, they are included here. There is sound advice on tumour grading but the statement that cytological examination of a tumour can give a definitive diagnosis may not meet with agreement of most neuropathologists who traditionally use smears for provisional diagnosis, depending on routinely processed sections for the definitive report.

The second part of the book gives a detailed and beautifully illustrated account of a wide range of diseases of peripheral nerve. The third section is an extensive description of muscle pathology paying particular attention to clinical correlation. Not many people have experience in ocular pathology but the detailed introduction in section four would give most pathologists the confidence to have a go. Subsequent sections give a lively account of ocular pathology.

The book is a worthy member of the Systemic Pathology Series, being easy to use for reference (despite the occasional very irritating internal reference within the index) and with illustrations of excellent standard. The book would be highly recommended for any department of histopathology or neuroscience, and at less than $£ 100$ might even be within the reach of the individual trainee in histopathology.

WANEY SQUIER
Intracranial Aneurysm Surgery: Techniques By DS SAMSON AND HH BATJER. (Pp 248; \$57.00). New York, Futura Publishing Co, 1990. ISBN 0-87993-3711.

It is impossible not to enjoy this book. It is written by two relatively young neurosurgeons who have devoted a great deal of time and attention to the study of vascular diseases of the nervous system and whose enthusiasm shows forth on every page. They have drawn liberally on experiences of other authors most of which they have referenced, although the European surgeon might with justification wonder why so few of the references pertain to other than the American literature.

There are of course always circumstances to which exception can be taken. Thus the authors recommend the use of CT with and without contrast in the initial management of subarachnoid haemorrhage cases, but do not justify their decision to give contrast. This is sometimes thought to carry certain risks to patients with impaired blood brain barrier permeability in the early stages following subarachnoid haemorrhage, and its only potential justification would be to outline an aneurysm prior to angiography. Angiography itself being mandatory, CT scanning with contrast seems superfluous.

Their description of individual aneurysm approaches is meticulous and detailed. Surprisingly perhaps, they have the exaggerated respect for Sylvian veins which categorises a number of American surgeons, and the ease of access to the anterior circle provided by routine sectioning of these vessels (in the author's experience always without sequelae provided there is a reasonable Labbe's vein seen on angiography) is ignored. The reviewer particularly enjoyed their detailed description of drilling of the clinoid, and their careful annotation of the treacherous relationship of the recurrent artery of Heubner to the medial aspect of terminal carotid aneurysms.

In regard to the basilar circulation, of course their experience is necessarily dwarfed by the enormous experience of Charles Drake, but the only caveat is that the advantages of the combined supra and infratentorial approach for giant upper and mid basilar aneurysms deserves a mention, as does the utility of a small anterior temporal resection in the access to very large upper basilar aneurysms. It should be of interest to all American surgeons that the approach to middle cerebral aneurysms through the superior temporal gyrus was first developed in the United Kingdom, before being popularised by Heros and others.

The authors almost rigid adherence to a right sided approach to anterior communicating aneurysms is one which cannot be recommended. The easiest approach to any aneurysm is the approach which most readily reaches the neck, and if the aneurysm arises from the proximal $\mathrm{ACl} /$ communicating junction on the left side, a right sided approach will reach its fundus before its neck, and as a result inevitably will be more difficult.

Regrettably the book does contain a number of serious distortions of the language. The verb "to mandate" is not a particularly attractive one, there can never (unless one is a politician) be "several alternatives", and the plural of "atheroma" one suspects should be atheroma rather than atheromata. The latter presumably is a neologism for atheromatous plaques. It would be churlish to suggest that the book would benefit from being translated 